\title{
Evaluating the role of OCT in optic disc analysis in glaucoma patients
}

\author{
AbdElmongy E. Ali, Abdullah M. EL-Amin, TagEldin M. Othman, Ahmed F. Gabr, Nesma O. \\ Mohamed
}

\author{
Department of Ophthalmology, Faculty of Medicine, Aswan University, Aswan, Egypt \\ Corresponding author: Nesma O. Mohamed, Mobile: (+20)01061199305, E-mail: drnesoo@yahoo.com
}

\begin{abstract}
Background: thirty patients underwent full ophthalmic examination, including a review of medical history, best-corrected visual acuity, slit-lamp biomicroscopy, intraocular pressure measurement, gonioscopy, dilated fundoscopic examination, automated perimetry, and OCT at 1 month and after 6 months.

Objectives: the aim of the study was to assess RNFL thickness, ONH clinically and by OCT and correlate RNFL thickness by OCT with visual fields by standard automated perimetry in glaucoma patients, highlighting the usefulness of OCT in glaucoma patients where diagnosis by standard means were not confirmatory.

Patients and methods: All patients were scanned using SD-OCT (RS-3000; Nidek) after dilation with 1\% tropicamide eye drop and the images were acquired by a single operator. Also, standard automated perimetry was conducted using the SITA 24-2 of the Humphrey Field Analyzer program (Carl Zeiss Meditec) with a Goldmann size III stimulus on a 31.5-apostilb background.

Results: There was a statistically significant difference between patients at 1 month and after 6 months as regards OCT (RNFL), on comparison between the four quadrants at 1 month the mean of the inferior quadrant was $>$ superior > temporal > nasal respectively. After 6 months the mean of the four quadrants as well as the total RNFL thickness decreased but only the decrease in the temporal quadrant and total RNFL thickness was statistically significant $(\mathrm{P}=0.003, \mathrm{P}=0.044$ respectively).

Conclusion: An OCT would be significantly informative early in the disease course to diagnose a preperimetric glaucoma, to confirm on a visual field, and to follow up glaucoma progression.
\end{abstract}

Keywords: Glaucoma, OCT

\section{Introduction}

Glaucoma is a leading cause of irreversible blindness throughout the world. A 1996 study estimated the prevalence of glaucoma in the world by the year 2000 to be 66.8 million, with 6.7 million suffering from bilateral blindness ${ }^{(\mathbf{1})}$.

Glaucoma is currently defined as a disturbance of the structural or functional integrity of the optic nerve that causes characteristic pathological changes, which may also lead to visual field defects over time. This disturbance usually can be minimized by adequate lowering of IOP and enhancement of optic nerve head (ONH) circulation. Primary open-angle glaucoma (POAG) is described distinctly as a multifactorial chronic and progressive optic neuropathy with a characteristic acquired loss of optic nerve fibers. Such loss develops in the presence of open anterior chamber angle, Characteristic visual field abnormalities, and elevated IOP that affects haemodynamic circulation of $\mathrm{ONH}$ and axoplasmic transport. POAG is a major worldwide health concern, because it has an insidious onset and leads to irreversible blindness ${ }^{(2)}$.

In glaucoma there is a relatively slow loss of retinal ganglion-cell axons. Early loss is usually in the mid-peripheral visual field. The disease becomes symptomatic at a relatively late stage when central vision is affected and the visual acuity declines or extensive loss of peripheral vision occur. Blindness due to glaucoma is largely can be minimized by early glaucoma discovery. While the visual damage is irreversible, it can usually be arrested, and to achieve this, early diagnosis have to be attempted ${ }^{(3)}$.

A number of ocular imaging devices have been introduced which provide reproducible quantification of the retinal nerve fiber layer (RNFL) and optic nerve head $(\mathrm{ONH})$. The commercially available 
glaucoma imaging devices include: optical coherence tomography (OCT), confocal scanning laser ophthalmoscopy (CLSO) and scanning laser polarimetry (SLP) $)^{(\mathbf{4})}$.

OCT is a non-invasive, noncontact, transpupillary, in vivo imaging system, using a super luminescent diode light source to create high resolution, real time, cross sectional tomographic images of retina. OCT is an interferometric imaging technique offering millimeter penetration depths of a sample under test with 10 micrometer axial and lateral resolution ${ }^{(4)}$.

\section{Aim of the Work:}

The aim of the study is to assess RNFL thickness, ONH clinically and by OCT and correlate RNFL thickness by OCT with visual fields by standard automated perimetryin glaucoma patients, highlighting the usefulness of OCT in glaucoma patients where diagnosis by standard means were not confirmatory.

\section{Patients and Methods}

\section{Inclusion criteria:}

Patients diagnosed as glaucoma or glaucoma suspect and fulfilling the following criteria were eligible for inclusion in the study;

- A high IOP greater than $21 \mathrm{mmHg}$ with $\mathrm{C} / \mathrm{D}$ ratio more than 0.4 , followed up by phasing.

- Glaucomatous visual field defects.

- Asymmetry of $\mathrm{C} / \mathrm{D}$ ratio between the two optic discs 0.2 ormore.

- Glaucomatous optic disc changes.

\section{Exclusion criteria:}

The following patients were excluded from the study;

- Patients with opaque media.

- Patients with visual acuity (V.A) less than $3 / 60$.

- Patients with other ocular pathologies that can cause secondary glaucoma or retinal nerve fiber layer (RNFL) disease.

- Patients with other major retinal orneural pathologies.
- Patients unsuitable for visual field testing or OCT imaging.

Methods:

Patients underwent full ophthalmic examination, including a review of medical history, best-corrected visual acuity, slit-lamp biomicroscopy, intraocular pressure measurement, gonioscopy, dilated fundoscopic examination, automated perimetry, and OCT at 1 month and after 6 months. Each eye was dilated with tropicamide $1 \%$ and scans was performed with a minimum pupillary diameter of $5 \mathrm{~mm}$.

\section{Instrumentation:}

\section{Standard Automated Perimetry}

Standard automated perimetry was conducted using the Swedish Interactive Threshold Algorithm (SITA) 24-2 of the Humphrey Field Analyzer program (Carl Zeiss Meditec) with a Goldmann size III stimulus on a 31.5-apostilb background. Qualified tests had a false positive and a false negative of $<20 \%$, and fixation losses of $<33 \%$. Healthy subjects had a glaucoma hemifield test (GHT) within normal limits and their mean deviation (MD) and pattern standard deviation (PSD) within $95 \%$ of healthy population. Glaucomatous VFs were defined as those with at least one of the following confirmed: GHT outside normal limits, or pattern standard deviation probability outside $95 \%$ of the healthy population.

\section{Spectral Domain Optical Coherence Tomography Imaging:}

All patients were scanned using SDOCT (RS-3000; Nidek). After dilation with $1 \%$ tropicamide eye drop and the images were acquired by a single operator.The subjects were asked to look at internal fixation target and a circular scan with a circle diameter of $3.4 \mathrm{~mm}$ was centered around optic disc. Average of three consecutive OCT images of the RNFL was obtained. The RNFL analysis uses an automated OCT software algorithm(Navis-EX version 1.4.1 (Nidek). The following RNFL parameters were evaluated: average peripapillary RNFLT $\left(360^{\circ}\right)$, the four quadrant average RNFLT (superior, nasal, inferior and temporal) and 12 
o'clock-hours RNFLT. The sectors were defined in clockwise order for the right eye and counter clockwise order for the left eye.

\section{Statistical analysis:}

The data was analyzed using IBM SPSS Statistics 24.0 program. Setting $\alpha=0.05$ and confidence interval 95\%. Screening for extreme values in quantitative variables was done using paired t-test. Discrete and categorical variables were screened using frequency distribution. Pearson correlation was used to determine the relation between RNFL thickness and other variables.

\section{Results}

Table (1): Demographic data of the study group

\begin{tabular}{|c|c|c|c|}
\hline \multirow{2}{*}{ Age } & Mean \pm SD & $49.87 \pm 13.04$ \\
\cline { 3 - 4 } & Male & Range & $23-70$ \\
\hline \multirow{2}{*}{ Sex } & Female & No (\%) & $15(50 \%)$ \\
\cline { 2 - 4 } & No (\%) & $15(50 \%)$ \\
\hline
\end{tabular}

As shown in table (1); regarding demographic data there were 15 male patients (50\%) and 15 female patients $(50 \%)$ whom age ranged from 23 to 70 years with mean of 49.87 .

Table (2): Comparison of clinical data of the study group at 1 month and after 6 months

\begin{tabular}{|c|c|c|c|c|c|}
\hline \multirow{2}{*}{\multicolumn{2}{|c|}{ variable }} & \multicolumn{2}{|c|}{ The studies cases $(n=60)$} & \multirow{4}{*}{$\begin{array}{c}\begin{array}{c}\text { Paired } \\
\text { t-test }\end{array} \\
2.615\end{array}$} & \multirow{4}{*}{\begin{tabular}{|} 
p-value \\
$0.011^{*}$
\end{tabular}} \\
\hline & & & & & \\
\hline \multirow{2}{*}{ BCVA } & Mean \pm SD & $0.788 \pm 0.229$ & $0.758 \pm 0.226$ & & \\
\hline & Range & $0.05-1.0$ & $0.05-1.0$ & & \\
\hline \multirow{2}{*}{ IOP } & Mean \pm SD & $16.11 \pm 3.004$ & $16.03 \pm 3.714$ & \multirow{2}{*}{0.160} & \multirow{2}{*}{0.874} \\
\hline & Range & $11-26$ & $11-28$ & & \\
\hline \multirow{2}{*}{ C/D } & Mean \pm SD & $0.705 \pm 0.151$ & $0.747 \pm 0.139$ & \multirow{2}{*}{-3.799} & \multirow{2}{*}{$0.001 * *$} \\
\hline & Range & $0.3-1.0$ & $0.3-1.0$ & & \\
\hline \multirow{2}{*}{ VF MD } & Mean \pm SD & $-7.73 \pm 7.599$ & $-7.67 \pm 8.30$ & \multirow{2}{*}{-0.136} & \multirow{2}{*}{0.893} \\
\hline & Range & $-32.52-0.69$ & $-32.05-0.74$ & & \\
\hline \multirow{2}{*}{ VF PSD } & Mean \pm SD & $4.52 \pm 3.455$ & $4.43 \pm 3.486$ & \multirow{2}{*}{304} & \multirow{2}{*}{0.763} \\
\hline & Range & $1.09-13.23$ & $1.09-13.04$ & & \\
\hline \multirow{2}{*}{ RNFL } & Mean \pm SD & $85.75 \pm 21.12$ & $80.68 \pm 17.28$ & \multirow{2}{*}{2.054} & \multirow{2}{*}{$0.044 *$} \\
\hline & Range & $37-145$ & $40-112$ & & \\
\hline
\end{tabular}

*significant $<0.05$.

As shown in table (2); regarding clinical data: on comparison between at 1 month and after 6 months there was statistically significant decrease in BCVA, RNFL ( $\mathrm{P}=0.011, \mathrm{P}=0.044$ respectively) and a highly statistically significant increase in $\mathrm{C} / \mathrm{D}$ ratio $(\mathrm{P}=0.000)$, While no statistically significant change occurred in IOP or VF.

Table (3): Comparison of RNFL thickness of the study group at 1month and after 6 months

\begin{tabular}{|c|c|c|c|c|c|}
\hline \multirow{2}{*}{\multicolumn{2}{|c|}{ RNFL }} & \multicolumn{2}{|c|}{ The studies cases $(n=60)$} & \multirow{2}{*}{$\begin{array}{c}\text { Paired } \\
\text { t-test }\end{array}$} & \multirow{2}{*}{ p-value } \\
\hline & & At 1month & After 6 months & & \\
\hline \multirow{2}{*}{ Inferior quadrant } & Mean \pm SD & $104.83 \pm 32.50$ & $98.78 \pm 30.80$ & \multirow{2}{*}{1.744} & \multirow{2}{*}{0.086} \\
\hline & Range & $46-195$ & $34-150$ & & \\
\hline \multirow{2}{*}{ Superior quadrant } & Mean \pm SD & $100.62 \pm 30.08$ & $96.43 \pm 24.19$ & \multirow{2}{*}{1.321} & \multirow{2}{*}{0.192} \\
\hline & Range & $11-155$ & $38-152$ & & \\
\hline \multirow{2}{*}{ Nasal quadrant } & Mean \pm SD & $68.2 \pm 23.82$ & $65.82 \pm 18.612$ & \multirow{2}{*}{0.831} & \multirow{2}{*}{0.409} \\
\hline & Range & $8-131$ & $10-109$ & & \\
\hline \multirow{2}{*}{ Temporal quadrant } & Mean \pm SD & $69.30 \pm 23.72$ & $58.78 \pm 14.77$ & \multirow{2}{*}{3.084} & \multirow{2}{*}{$0.003 *$} \\
\hline & Range & $4-192$ & $30-127$ & & \\
\hline \multirow{2}{*}{ Total } & Mean \pm SD & $85.75 \pm 21.12$ & $80.68 \pm 17.28$ & \multirow{2}{*}{2.054} & \multirow{2}{*}{$0.044^{*}$} \\
\hline & Range & $37-145$ & $40-112$ & & \\
\hline
\end{tabular}

As shown in table (3): Regarding RNFL thickness: on comparison between the four quadrants at 1month the mean of the inferior quadrant was $>$ superior $>$ temporal $>$ nasal $(104.83>100.62>68.58>68.2$ 
respectively).After 6 months the mean of the four quadrants as well as the total RNFL thickness decreased but only the decrease in the temporal quadrant and total RNFL thickness was statistically significant $(\mathrm{P}=0.003$, $\mathrm{P}=0.044$ respectively).

Table (4): Correlation between percentage of change of total RNFL thickness with demographic data, percentage of change of clinical data and four quadrants RNFL thickness

\begin{tabular}{|c|c|c|c|}
\hline Variable & Mean \pm SD & Pearson correlation & P-value \\
\hline Age & $49.87 \pm 13.039$ & 0.073 & 0.580 \\
\hline Sex & $50 \%$ males,50\% females & -.006 & 0.961 \\
\hline BCVA & $4.39 \pm 9.68$ & 0.138 & 0.294 \\
\hline IOP & $17.51 \pm 17.96$ & -0.025 & 0.851 \\
\hline C/D & $8.09 \pm 23.27$ & -0.029 & 0.827 \\
\hline VF MD & $536.50 \pm 2986.9$ & -0.093 & 0.480 \\
\hline VF PSD & $28.89 \pm 27.49$ & 0.083 & 0.526 \\
\hline RNFL & $16.04 \pm 19.80$ & 1 & - \\
\hline Inferior quadrant & $17.38 \pm 22.27$ & 0.657 & $0.000^{* *}$ \\
\hline Superior quadrant & $26.88 \pm 74.97$ & 0.635 & $0.001^{* *}$ \\
\hline Nasal quadrant & $34.42 \pm 79.13$ & 0.432 & $0.001^{* *}$ \\
\hline Temporal quadrant & $20.05 \pm 25.94$ & 0.526 & $0.001^{* *}$ \\
\hline
\end{tabular}

As shown in table (4): Regarding percentage of change of RNFL thickness there was no statistically significant correlation with demographic data or percentages of change of clinical data.On the other hand, the percentage of change of RNFL thickness had highly statistically significant correlation of the inferior, superior, nasal and temporal quadrants $(\mathrm{P}=0.001, \mathrm{P}=0.001$, $\mathrm{P}=0.001, \mathrm{P}=0.0001$ respectively)

\section{Discussion}

Glaucomatous damage can be quantified using either structural or functional loss criteria, or a combination of both. Patients with glaucoma may present with the disease before damage is detectable with standard achromatic automated perimetry (preperimetric glaucoma) or with clear glaucomatous visual field defects (perimetric glaucoma) ${ }^{(5)}$.

OCT is a developed, noninvasive, noncontact technique for imaging of the layered structure of the retina ${ }^{(6)}$.

Attempts to measure RNFL thickness in normal and glaucomatous eyes using OCT have been made by several investigators ${ }^{(7)}$. However, considerable inter-individual variations in RNFL thickness have been demonstrated even in normal individuals ${ }^{(\mathbf{8})}$.

Some studies suggested that OCT may be superior to other imaging techniques, such as scanning laser polarimetry and Heidelberg retina tomography, for detecting a specific pattern of reduction in the average or focal (RNFL) thickness ${ }^{(7)}$.

Our patients underwent a complete ophthalmological examination, visual field examination, and OCT at two visits with a 6month time interval. We found no association between the SD-OCT measurements and the demographic data of the subject. Similar results have been reported by different groups who evaluated this relationship in their measurements.

Schuman et $\boldsymbol{a l l}^{\left({ }^{(9)}\right)}$ showed that RNFL thickness of men were usually thinner than that of women but not statistically significant. Our results agree with Ramakrishnan et al. (10) and Rao et al. (11) that have shown no significant gender-related difference in RNFL thickness.

There was a statistically significant correlation between $\mathrm{C} / \mathrm{D}$ and average RNFL thickness in glaucoma patients. This is in agreement with the results of Manassakorn et $\boldsymbol{a l .} .^{(12)}$, as they found that there was a statistically significant difference in C/D between glaucoma patients at 1 month and after 6 months.

There was no statistically significant change occurred in VF despite of decrease in average RNFL thickness. Hood et al. (13) conducted a study with similar results. They reported that the superiority of OCT RNFL measurement in detection of early glaucoma could be related to the relative difference in 
standard deviations of OCT and visual field measurements.In the early stage of disease, more patients show significant RNFL loss and normal visual field results than those with significant visual field loss and normal RNFL measurement. For this reason, it is not surprising to observe a poor agreement between functional and structural tests in the evaluation of glaucoma progression.

Also, Soliman et al. ${ }^{(14)}$ and Kanamori et al. (15) conducted a study with similar results. They noted that OCT has the ability to detect glaucomatous changes by measuring RNFL thickness, particularly the average. Further, average and quadrant thickness had good correlation with the mean deviation on the Humphery field analyzer.

While our results are not in correlation

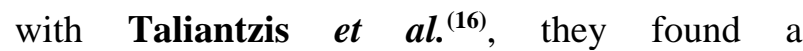
moderate correlation between RNFL thickness measured by Stratus OCT and VF indices (mean sensitivity, mean defect, loss variance). The correlation became stronger when the structural alterations became deeper in OCT.

Quigley et $\boldsymbol{a l} .{ }^{(\mathbf{1 7})}$ showed pre-existing RNFL defects in $57 \%$ of glaucoma suspect eyes that converted from normal to defective visual fields. Pre-existing RNFL defects were also present in $35 \%$ of nonconverted eyes. Moreover, the risk of conversion of defective visual fields in glaucoma suspect eyes increased with the increase in RNFL damage.

It seems that using visual field loss as the only endpoint in clinical trials limits the interpretation of the behavior of the disease, because it is a subjective test with large variability and limited range and requires longer follow-up time, and there is no consensus on the best method of judging progression.

In our study regarding RNFL thicknesson comparison between the four quadrants at 1 month the mean of the inferior quadrant was $>$ superior $>$ temporal $>$ nasal $(104.83>100.62>68.58>68.2$ respectively). This is in correlation with those of Kanamori et al. ${ }^{(15)}$.

Also, Sony et al. ${ }^{(18)}$ observed that the RNFL was thickest in the inferior and superior quadrants and the thickness was lesser in nasal and temporal quadrants.
After 6 months the mean of the four quadrants as well as the total RNFL thickness decreased but only the decrease in the temporal quadrant and total RNFL thickness was statistically significant.

While, Leung et al. ${ }^{(19)}$ analysis of the pattern of RNFL defects with SD-OCT imaging as they demonstrated that the most frequently RNFL defects have been at the inferotemporal meridian followed by the superotemporal meridian.

In our study RNFL defect extended through almost all sectors in the advanced disease subgroups as noted by Manassakorn et al. ${ }^{(20)}$.

Also, Budenz et al. (21) studies showed that both the measurement and classification agreements between the consecutive scans were lower in the nasal quadrant than in the other quadrants.

However, our results are not in agreement with those of Mwanza et al..$^{(22)}$ who reported that, in the mild primary open angle glaucoma patients, focal RNFL thickness loss was found in the inferior area.

It should be noted that changes may occur on different sectors of the optic disc and RNFL as glaucoma progresses, which could explain why a single parameter may not be able to detect all progressing eyes ${ }^{(\mathbf{2 3})}$.

The use of SD OCTs is likely to overcome this limitation and result in improved reproducibility of RNFL measurements. However, the ability of these instruments to detect progressive glaucomatous damage has not yet been evaluated ${ }^{(24)}$.

Previous studies have found OCT ONH parameters to be reproducible and to perform well in discriminating eyes with glaucomatous visual field loss from healthy eyes. However, in the present study, we found that these parameters had poor ability to differentiate progressing from nonprogressing eyes ${ }^{(25)}$.

\section{Conclusion}

Early diagnosis and treatment of glaucoma can prevent vision loss from that disease. Ophthalmologists play an important role in the diagnosis of glaucoma by following up patients with 
positive family history or with suspicious optic nerve head findings. They can improve treatment outcomes by reinforcing the importance of medication adherence and persistence and by recognizing adverse reactions from glaucoma medications and surgeries.

They calculated that screening would decrease the prevalence of glaucoma from $75 \%$ to $38 \%$, reduce the prevalence of severe vision loss from $29.1 \%$ to $23.9 \%$, and double the diagnosis of mild visual field loss from $9.2 \%$ to $18.7 \%$.

Measuring the rate of RNFL thickness loss would be important in discerning the course of glaucoma progression, prediction of disease prognosis, and evaluation of treatment response.

Optical coherence tomography has changed the face of glaucoma assessment and research. OCT can provide objective, quantitative, and reproducible images of the ONH and RNFL, both of which undergo structural changes in glaucoma before visual field loss. Spectral domain OCT provides three-dimensional video imaging of the $\mathrm{ONH}$, three-dimensional RNFL thickness maps, and optical Doppler tomography.

An OCT would be significantly informative early in the disease course to diagnose a preperimetric glaucoma, to confirm on a visual field, and to follow up glaucoma progression.

However, the evaluation by SD-OCT is still not superior to ophthalmologist. Clinical decisions should never be driven by OCT results alone, but should also be based on a complete ophthalmic examination and VF assessment.

\section{References}

1. Quigley HA (1996): Number of people with glaucoma worldwide. British Journal of Ophthalmology, 80:389-391.

2. Jerald AB (1998): Changing definition of glaucoma. Am. J. Ophthalmol., 7:165169.

3. Goldberg I, Graham SL and Healey PR (2002): Primary open-angle glaucoma. Med. J. Aust., 177:535-536.

4. Diana J, Ramesh K, Jayasree $\mathrm{S}$ et al. (2015): Endodontic management of a mandibular first molar withradix entomolaris- a case report. IOSR Journal of Dental and Medical Sciences, 14(7): 21-24.
5. Susanna R, Vessani RM (2009): Staging glaucoma patient: why and how? Open Ophthalmol. J., 3:59- 64.

6. Hee MR, Swanson EA and Burr J (1995): Optical coherence tomography of the human retina. Arch. Ophthalmol., 113:325-332.

7. Bowd C, Zangwill LM, Berry CC et al. (2001): Detecting early glaucoma by assessment of retinal nerve fiber layer thickness and visual function. Invest. Ophthalmol. Vis. Sci., 42:1993-2003.

8. Repka MX, Quigley HA (1989): The effect of age on normal human optic nerve fiber number and diameter. Ophthalmology, 96:26-32.

9. Schuman JS, Pedut-Kloizman T, Hertzmark E et al. (1996): Reproducibility of nerve fiber layer thickness measurements using optical coherence tomography. Ophthalmology, 103:1889-1898.

10. Ramakrishnan R, Mittal S, Ambatkar $S$ et al. (2006): Retinal nerve fiber layer thickness measurements in normal Indian population by optical coherence tomography. Indian J. Ophthalmol., 54:11-15.

11. Rao HL, Kumar AU, Babu JG et al. (2011): Predictors of normal optic nerve head, retinal nerve fiber layer, and macular parameters measured by spectral domain optical coherence tomography. Invest. Ophthalmol. Vis. Sci., 52:11031110 .

12. Manassakorn A, Nouri-Mahdavi $K$ and Caprioli J (2006): Comparison of retinal nerve fiber layer thickness and optic disk algorithms with optical coherence tomography to detect glaucoma. Am. J. Ophthalmol., 141:105115 .

13. Hood DC, Kardon RH (2007): A framework for comparing structural and functional measures of glaucomatous damage. Prog. Retin. Eye Res., 26: 688710.

14. Soliman MA, Van Den Berg TJ, Ismaeil AA et al. (2002): Retinal nerve fiber layer analysis: relationship between 
optical coherence tomography and redfree photography. Am. J. Ophthalmol., 133:187- 195.

15. Kanamori A, Nakamura $M$, Escano MF et al. (2003): Evaluation of the glaucomatous damage on retinal nerve fiber layer thickness measured by optical coherence tomography. Am. J. Ophthalmol., 135:513-520.

16. Taliantzis S, Papaconstantinou D, Koutsandrea $\mathrm{C}$ et al. (2009): Comparative studies of RNFL thickness measured by OCT with global index of visual fields in patients with ocular hypertension and early open angle glaucoma. Clin. Ophthalmol., 3: 373379.

17. Quigley HA, Enger C, Sommer A et al. (1994): Risk factors for the development of glaucomatous visual field loss in ocular hypertension. Arch. Ophthalmol., 112 (1994): 644-649.

18. Sony $P$, Sihota $R$, Tewari HK et al. (2004): Quantification of the retinal nerve fibre layer thickness in normal Indian eyes with optical coherence tomography. Indian J. Ophthalmol., 52(4):303-309.

19. Leung CK, Choi $\mathrm{N}$, Weinreb $\mathrm{RN}$ et al. (2010): Retinal nerve fiber layer imaging with spectral-domain optical coherence tomography: pattern of RNFL defects in glaucoma. Ophthalmology, 117: 23372344.
20. Manassakorn A, Aupapong S (2011): Retinal nerve fiber layer defect patterns in primary angle-closure and open-angle glaucoma: a comparison using optical coherence tomography. Japn. J. Ophthalmol., 55: 28-34.

21. Budenz DL, Fredette MJ, Feuer WJ et al. (2008): Reproducibility of peripapillary retinal nerve fiber thickness measurements with Stratus OCT in glaucomatous eyes. Ophthalmology, 115: 661-666.

22. Mwanza JC, Chang RT, Budenz DL et al. (2010): Reproducibility of peripapillary retinal nerve fiber layer thickness and optic nerve head parameters measured with cirrus HDOCT in glaucomatous eyes. Invest. Ophthalmol. Vis. Sci., 51: 5724-5730.

23. Vizzeri G, Bowd C, Medeiros FA et al. (2009): Scan tracking coordinates for improved centering of Stratus OCT scan pattern. J. Glaucoma, 18:81 -87.

24. Gonzalez-Garcia AO, Vizzeri G, Bowd C et al. (2009): Reproducibility of RTVue retinal nerve fiber layer thickness and optic disc measurements and agreement with Stratus optical coherence tomography measurements. Am. J. Ophthalmol., 147:10-67.

25. Leung CK, Cheung CY, Lin D et al. (2008): Longitudinal variability of optic disc and retinal nerve fiber layer measurements. Invest. Ophthalmol. Vis. Sci., 49:4886-48 92. 